\title{
A Rare Huge Sacral Tarlov Cyst with Progressive Neurologic Deficit: A Case Report
}

\author{
Kaveh Haddadi ${ }^{1}$, Hamid Reza Ganjeh Qazvini ${ }^{2}$, Hassan Shayan Majd ${ }^{3}$, Mojtaba Saleh Ahangar ${ }^{4}$ \\ ${ }^{1}$ MD, Assistant Professor, Department of Neurosurgery, Emam Hospital, Orthopedic Research Center, Mazandaran University of Medical Sciences, \\ Sari, Iran \\ ${ }^{2}$ MD, Resident of Neurosurgery, Department of Neurosurgery, Faculty of Medicine, Mazandaran University of Medical Sciences, Sari, Iran \\ ${ }^{3} \mathrm{MD}$, Resident of Neurosurgery, Department of Neurosurgery, Faculty of Medicine, Mazandaran University of Medical Sciences, Sari, Iran \\ ${ }^{4} \mathrm{MD}$, Resident of Neurosurgery, Department of Neurosurgery, Faculty of Medicine, Mazandaran University of Medical Sciences, Sari, Iran \\ * Corresponding Author Address: Orthopedic Research Center, Department of Neurosurgery, Emam Hospital, Mazandaran University of Medical \\ Sciences, Sari, Iran. Tel: +981133378789, Fax:+981133378789. E-mail: kh568hd@yahoo.com
}

Article Type: Case Report

Received: May 14, 2016, Last Revised: September 2, 2016 , Accepted: September 12, 2016

\section{Abstract}

Background and Importance: Perineural cysts, also known as Tarlov cysts, are benign cysts of the spinal cord containing a collection of cerebrospinal fluid. They most frequently originate in the sacral spine and can be asymptomatic.

Case Presentation: We presented a 30-year-old woman who was referred to our clinic with an 8-month history of perianal paresthesia, slowly progressive lower back pain, pain in back of the left and right thigh, and a one-week history of bladder incontinence. Magnetic resonance imaging (MRI) done six months ago showed a large cyst with size of 74x40x22 mm in S1 from S4 of the spinal canal. The cyst was uncovered after laminectomy of S1 to S4. The cyst was aspirated, its neck ligated, and the dismissed portion was excised. The nerve roots were reserved intact. Postoperatively, the patient reported noticeable pain relief. At month 3 , the patient's bowel and bladder control recovered. Sensation and deep reflexes were also normal.

Conclusion: A giant sacral Tarlov cyst and the development of associated radicular symptoms are powerfully associated with an outstanding outcome following surgery.

Keywords: Huge; Tarlove Cyst; Sacral; Neurologic Deficit

Please cite this paper as: Haddadi K, Ganjeh Qazvini H.R, Shayan Majd H, Saleh Ahangar M. A Rare Huge Sacral Tarlov Cyst with Progressive Neurologic Deficit: A Case Report. Iran. J. Neurosurg. 2016;2(2):30-33

\section{Background and Importance}

Perineural cysts, well known as Tarlov cysts originated from the name of the first author who described them in 1938 as additional definitions throughout autopsy, are benign cysts of the spinal cord containing cerebrospinal fluid (13). While there are regularly multiple cysts originated from dorsal nerve root that are nearly always observed in the spinal cord, they most frequently originate in the sacral spine asymptomatically $(8,9,14)$. MRI is presently the gold standard modality for revealing such cysts (7). The clinical appearance of symptomatic Tarlov cyst is non-specific, and can mimic other spinal complaints: localized pain, radiculopathy, sensory disturbance, weakness, and bladder and bowel dysfunction $(7,13,14)$. Although operating interventions have been established for handling Tarlov cysts (13-15), the conservative method is clinically favorite as a means of avoiding invasive surgery. Some clinicians have reported good consequences resulting from the use of steroid therapy $(2,11)$. After reviewing the available literature, we found that our patient had the biggest Tarlov cyst among the cases ever recorded $(4-6,12,13,16)$.

\section{Case Presentation}

\section{History}

In September 2015, a 30-year-old woman referred to our clinic with 8-month history of perianal paresthesia, slowly progressive pain in the lower back and in the back of the left and right thigh, and a one-week history of bladder incontinence.

\section{Examination}

Examination revealed a diminished ankle jerk on the right side and hypoesthesia in S2 to S4 dermatomes. Straight leg raising was restricted on the right side, and she had plantar dorsi flexion weakness of about $3 / 5$ in the right foot. She was supported for about six months by one of our colleagues using a conservative treatment, with a diagnosis of spinal cord cyst. MRI undertaken six months before treatment showed a large cyst with size of $74 \times 40 \times 22 \mathrm{~mm}$ in S1 from S4 of the spinal canal extending into the neural foramina, causing an impingement of the exiting and traversing nerve fibers (Figures 1,2,3). This was comparable to the lesion that discovered in a new repeated MRI. CT scan showed an extensive bone erosion in the cyst site in sagittal view (Figure 4). An electrodiagnostic test indicated severe and chronic irritability of all the lower lumbosacral spinal roots.

\section{Operation}

The cyst was uncovered after laminectomy of S1 to S4 in prone position. It was situated near the dorsal root ganglion of S2 and S3. It was aspirated and was discovered to be filled with cerebrospinal fluid; the Valsalva maneuver was used for approval. Both motor and sensory fibers appeared to be attached to the cyst wall. The cyst was aspirated, its neck ligated, and the unnecessary portion was excised. The nerve root was reserved intact. No inflow of cerebrospinal fluid was apparent during a repeated Valsalva maneuver. We undertook a detailed dural repair and used fat and fibrin glue for the prevention of a CSF leak after surgery (Figure 5,6). 

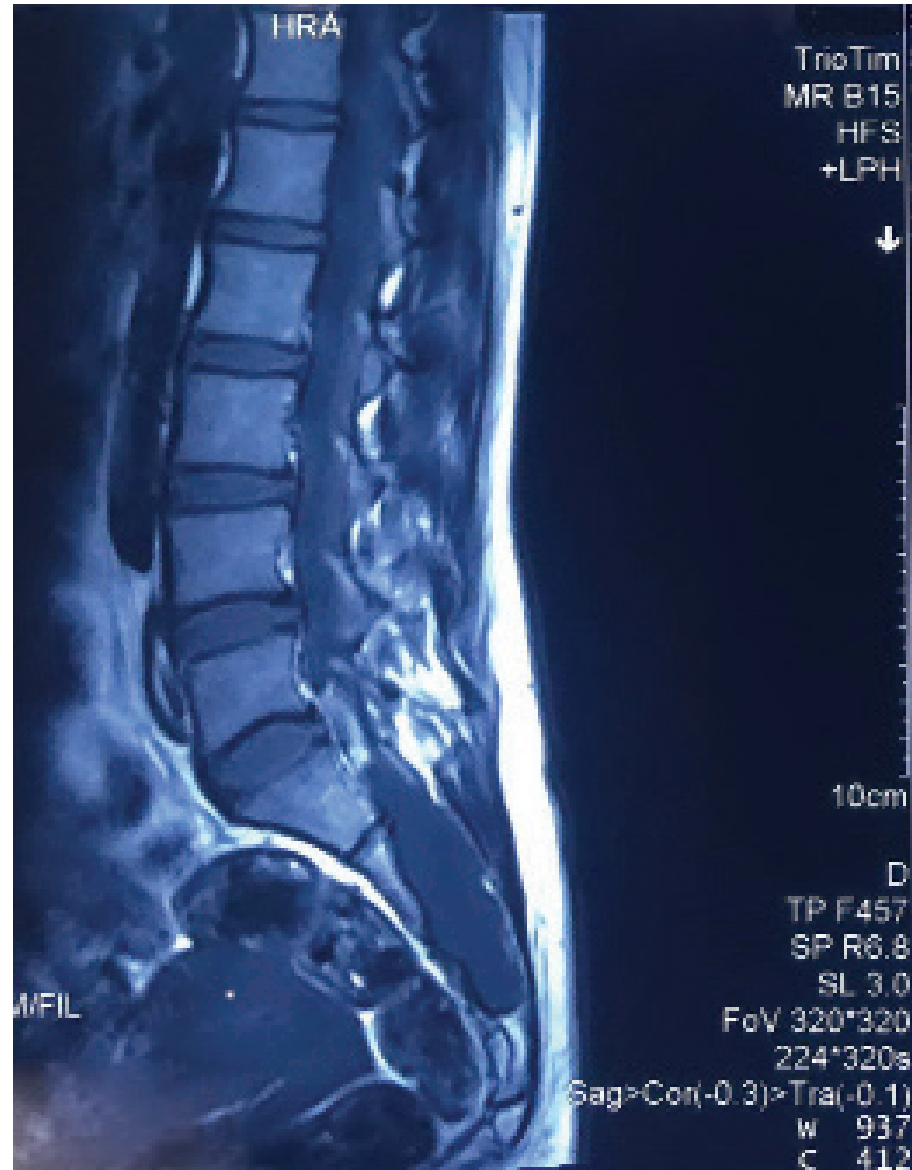

Figure 1. Sagittal T1 MRI; Revealing a Large Cyst in S1 from S4 of the Spinal Canal Extending into the Neural Foramina, Causing Thecal Sac Compression

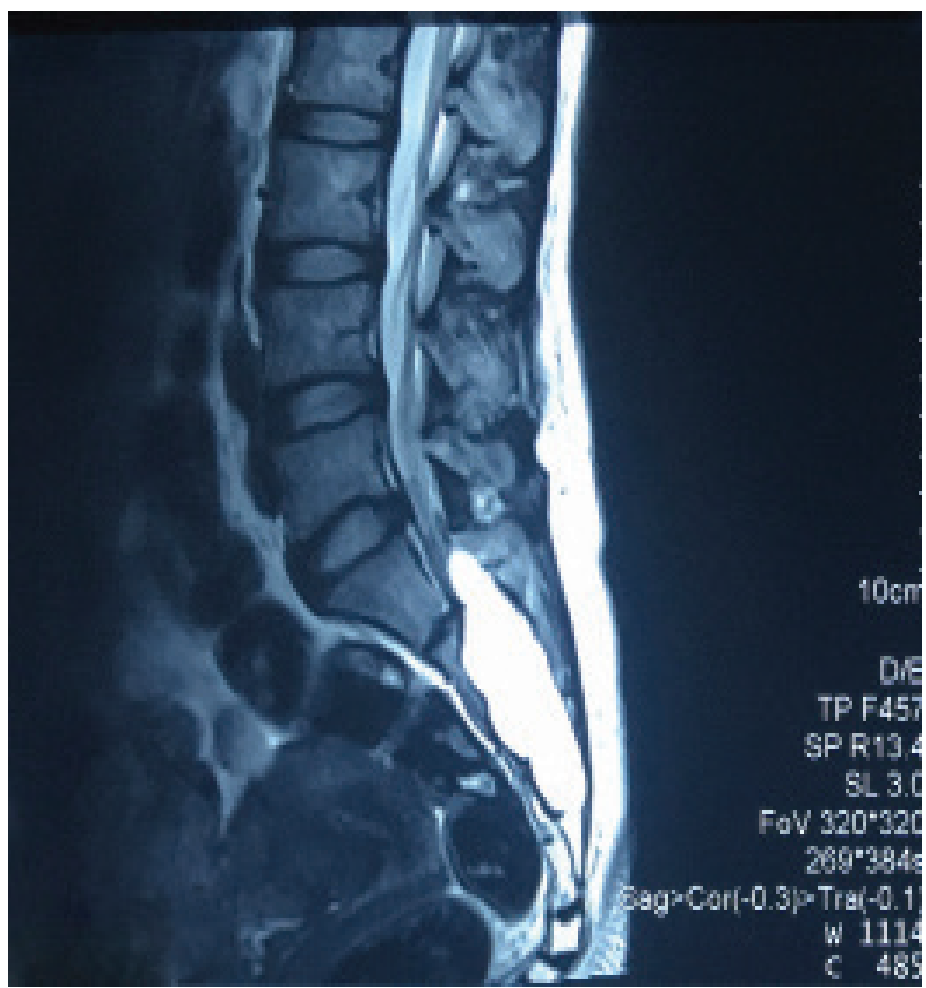

Figure 2. Sagittal T1 MRI; Revealing a Large Cyst Measuring in S1 from S4 of the Spinal Canal Extending into the Neural Foramina, Causing Thecal Sac Compression

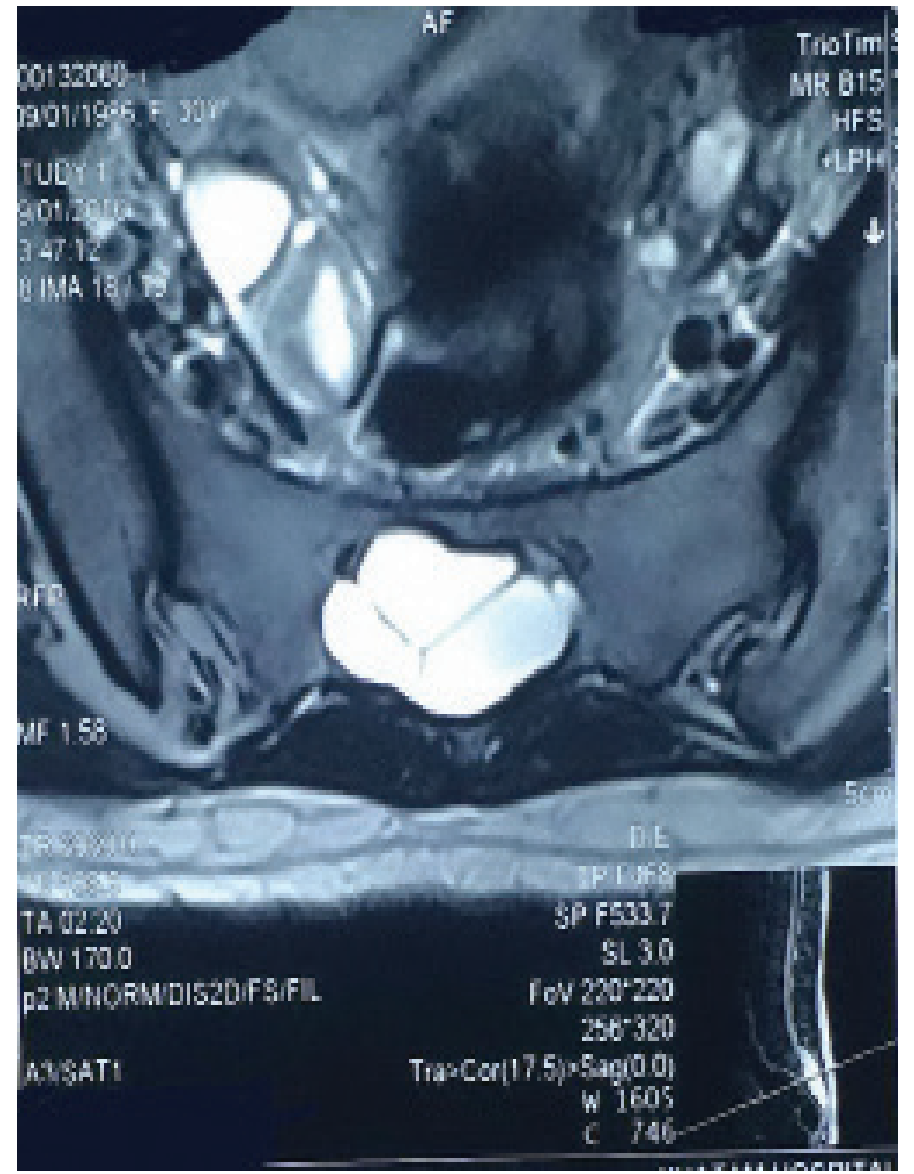

Figure 3. Axial MRI; Revealing a Large Cyst in Sacral Area

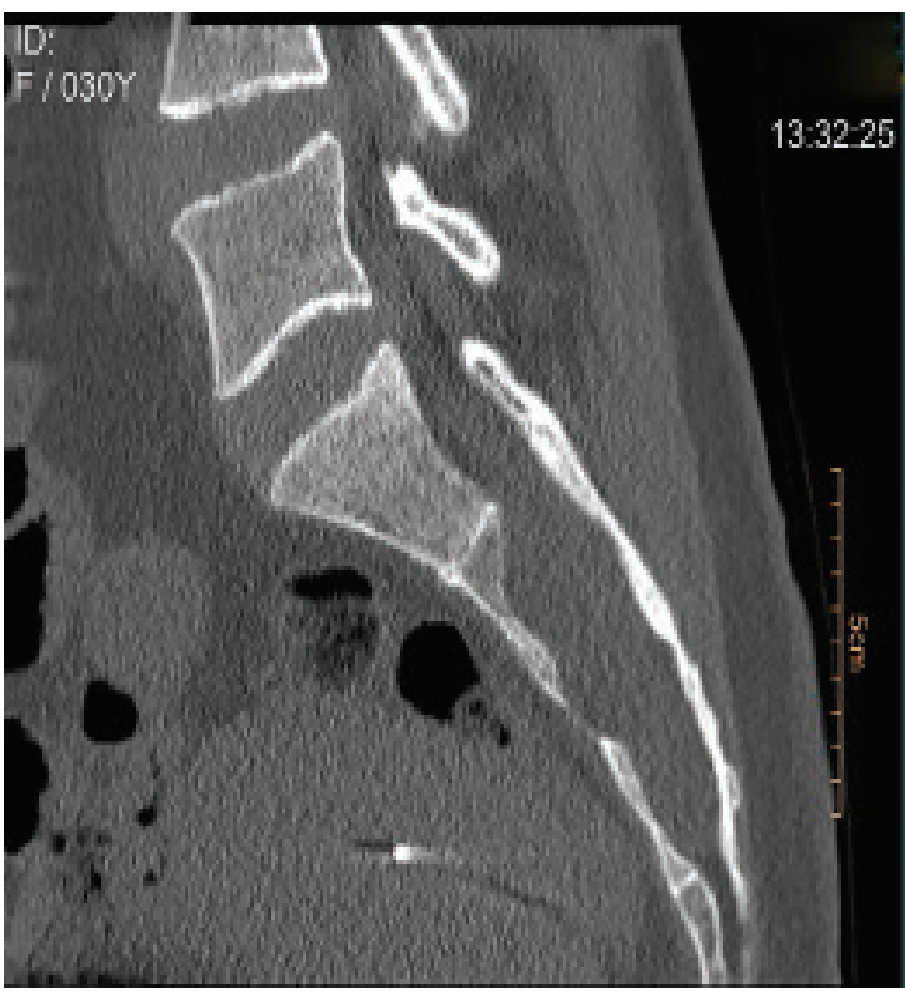

Figure 4. A CT Scan; Showing Extensive Bone Erosion in the Cyst Site in Sagittal View 


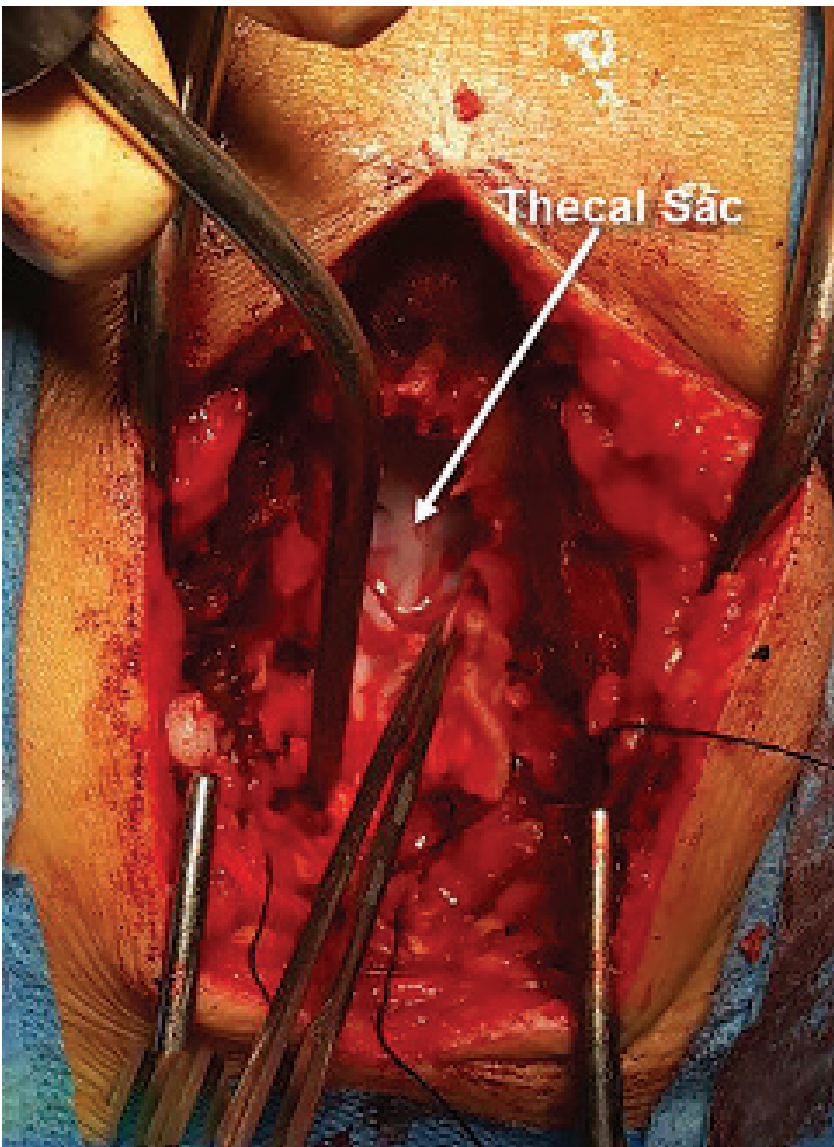

Figure 5. Decompressed Cyst Space in Operative Field

\section{Postoperative Course}

Postoperatively, the patient reported noticeable pain relief. By third, sixth and eleventh month, bowel and bladder control recovered. Sensation and deep reflexes were also normal. At the present time, the patient is neurologically intact and symptom-free. It should be mentioned that written informed consent was obtained from the patient for publication of this case report and accompanying images.

\section{Discussion}

Perineural cysts are formed in the perineural space among the endoneurium derived from the pia matter and the perineurium shaped by the arachnoidal matter (1). They happen lengthwise along the nerve ganglion (1). Their walls contain nerve fibers and/or ganglion cells (10). They have been referred to as "...extradural meningeal cysts with nerve root fibers" (type II) and, sometimes they are supposed to connect with the subarachnoid space, although some authors oppose it $(2,14)$. They most frequently originate in the sacral spine with an occurrence of $4.6 \%$ in a symptomatic populace based on a retrospective evaluation of MRIs of the lumbosacral spine (8).

Rupture of a Tarlov cyst, infrequent as it might be, should be measured in patients with cerebral fat embolisms, particularly after lower back trauma (15). In the past, the ball-valve mechanismhas been assumed to be the cause of why some large Tarlov cysts develop increased symptoms, while others give rise to only mild symptoms (11). The beginning of such symptoms can be rapid or gradual. Typically, patients report that their symptoms are worsened by standing, coughing, and

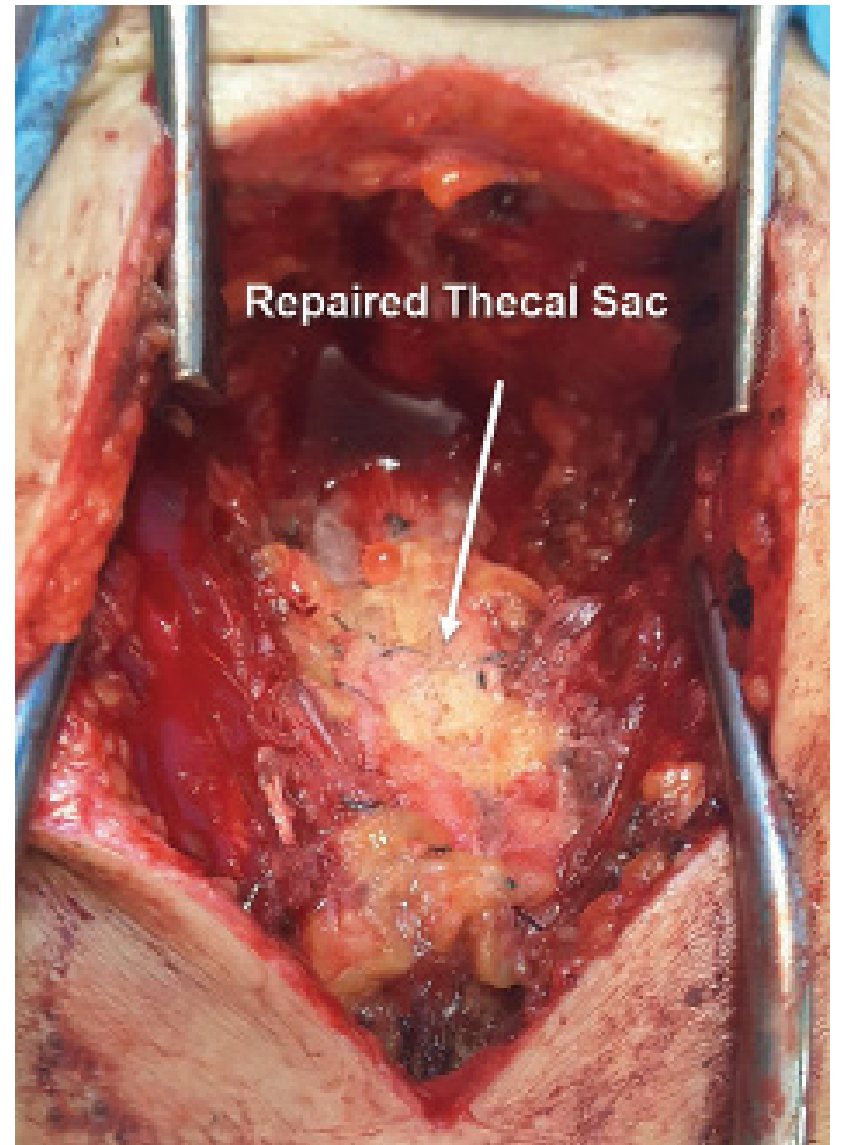

Figure 6. Repaired Operative Field with Fat and Fibrin Glue after Cyst Resection for Preventation of CSF Leak

by a change of position. This can be clarified by the rise in CSF pressure, leading to an initiation of the above-mentioned ball-valve mechanism. Symptomatic release can regularly be attained by horizontal positioning (11).

MRI is now the technique of choice in finding perineural cysts. The indications that support the diagnosis of a perineural cyst are the demonstration of a low signal in terms of T-1 weighted images, and a high signal with regard to T-2 weighted images, comparable to CSF (11), as well as shape and the neighborhood of its location to the dorsal ganglion (2). Since Tarlov cysts are frequently incidental, their finding can lead to three dissimilar diagnostic possibilities: 1) additional pathology is producing symptoms; 2) additional pathology is perhaps causing symptoms, with the Tarlov cyst possibly being a secondary reason for the symptoms; or 3) the Tarlov cyst is the only pathological finding that can indicate the symptoms. Clearly, there is a need to prudently assess the association between clinical and radiological results (7). The surgical options are: 1) diversion of the CSF flow (CT-guided percutaneous aspiration, lumboperitoneal shunt); and 2) direct microsurgical methods (cyst fenestration, cyst neck ligation, cyst resection, and combinations of the above) (11). Each option comes with the possibility of serious complications such as cyst recurrence and aseptic meningitis.

Lumbar cerebrospinal fluid drainage in conjunction with a lumboperitoneal shunting process and cyst subarachnoid shunt application are new treatment options. In multiple Tarlov cysts cases, these treatment options should be useful.

After a long period of failure in terms of the use of a 
conservative therapy, the patient was referred to the surgical team. Given the severity of the symptoms, we decided to treat her pathology as soon as possible and perform emergency surgery after reviewing the available literature. We found that our patient was the biggest Tarlov cyst case ever recorded $(4-6,12,13,16)$.

\section{Conclusion}

We can assume that the surgical treatment of Tarlov cysts is optional if symptomatic cases are selected suitably. Giant sacral Tarlov cysts in conjunction with associated radicular symptoms have powerfully relationship with outstanding outcomes following surgery.

\section{Funding}

None.

\section{Conflicts of Interest}

The authors declare no conflicts of interest.

\section{Authors' Contribution}

Conception and design: Kaveh Haddadi, Hamid Reza Ganjeh Qazvini. Data Collection: Kaveh Haddadi, Hamid Reza Ganjeh Qazvini. Drafting the article: Hamid Reza Ganjeh Qazvini, Mojtaba Saleh Ahangar. Critically revising the article: Kaveh Haddadi, Hassan Shayan Majd. Reviewed submitted version of manuscript: all authors. Approved the final version of the manuscript: all authors.

\section{References}

1. Bartels RH, van Overbeeke JJ. Lumbar cerebrospinal fluid drainage for symptomatic sacral nerve root cysts: an adjuvantdiagnostic procedure and/or alternative treatment? Technical case report. Neurosurgery 1997; 40:861-5.

2.Caspar W, Papavero L, Nabhan A, Loew C, Ahlhelm F. Microsurgical excision of symptomatic sacral perineurial cysts: astudy of 15 cases. Surg Neurol 2003; 59:101-6.

3. Geert J. Vermeersch, Paul M. Vanderschot. Transverse sacral fracture through Tarlov cyst causing a cauda equina syndrome:Case report and review of the literature. Injury Extra 40:270-273; 2009.

4. Hakan Kayali, B Lent D Z, Eng N G N L. Symptomatic Sacral Tarlov Cyst: Case Report. Journal of Ankara Medical School; 25(2): 103-106; 2003.

5. Jayesh P Thawani, Hovik John Ashchyan, John Pierce,etal. Microsurgical Treatment of a Complex Sacral Perineural Cyst Using Paraspinous Muscle Flap: Technical Note. J Spine Neurosurg 2015, 4:2

6. Kwan Leung Chia. Symptomatic Tarlov cyst and electroacupuncture: more studies required. J Integr Med.2015; 13(1): 58-60.

7. Lucantoni C, Than KD, Wang AC, Valdivia-Valdivia JM, Maher CO, et al. (2011) Tarlov cysts: a controversial lesion of the sacral spine. Neurosurg Focus 31: E14.

8. Nabors MW, Pait TG, Byrd EB, Karim NO, Davis DO, et al. (1988) Updated assessment and current classification of spinal meningeal cysts. J Neurosurg 68: 366-377.

9. Paulsen RD, Call GA, Murtagh FR (1994) Prevalence and percutaneous drainage of cysts of the sacral nerve root sheath (Tarlov cysts). AJNR Am JNeuroradiol 15: 293-297.

10. Paulsen RD, Call GA, Murtagh FR. Prevalence and percutaneous drainage of cysts of the sacral nerve root sheath (Tarlovcysts). AJNR Am J Neuroradiol 1994; 15:293-9.

11. Patel MR, Louie W, Rachlin J. Percutaneous fibrin glue therapy of meningeal cysts of the sacral spine. AJR Am J Roentgenol1997; 168:367-70.

12. Ramesh Kumar Sen, Tarun Goyal, Sujit Kumar Tripathy, Soumya Chakraborty. Tarlov cysts: a report of two cases. Journal of Orthopaedic Surgery $2012 ; 20(1): 87-9$.

13. Tarlov IM (1938) Perineurial cysts of the spinal nerve roots. Arch NeurolPsychiat (Chic) 40: 1067-1074.

14. Tarlov IM (1970) Spinal perineurial and meningeal cysts. J Neurol Neurosurg Psychiatry 33: 833-843.

15. Voyadzis JM, Bhargava P, Henderson FC. Tarlov cysts: a study of 10 cases with review of the literature. J Neurosurg 2001; 95(1Suppl):S25-32.

16. Zibis H. Aristeidis, Fyllos C. Apostolos,Arvanitis L Dimitris. Symptomatic Cervical Perineural (Tarlov) Cyst: A Case Report. Clin Med Rev Case Rep 2015, 2:2. 